\title{
Generation of male germ cells from induced pluripotent stem cells (iPS cells): an in vitro and in vivo study
}

\author{
Yong Zhu ${ }^{1, *}$, Hong-Liang $\mathrm{Hu}^{1, *}$, Peng Li ${ }^{1}$, Shi Yang ${ }^{1}$, Wei Zhang ${ }^{1}$, Hui Ding ${ }^{1}$, Ru-Hui Tian ${ }^{1}$, Ye Ning ${ }^{1}$, \\ Ling-Ling Zhang ${ }^{2}$, Xi-Zhi Guo ${ }^{2}$, Zhan-Ping Shi ${ }^{1}$, Zheng $\mathrm{Li}^{1}$ and Zuping $\mathrm{He}^{3,4}$
}

Recent studies have reported that induced pluripotent stem (iPS) cells from mice and humans can differentiate into primordial germ cells. However, whether iPS cells are capable of producing male germ cells is not known. The objective of this study was to investigate the differentiation potential of mouse iPS cells into spermatogonial stem cells and late-stage male germ cells. We used an approach that combines in vitro differentiation and in vivo transplantation. Embryoid bodies (EBs) were obtained from iPS cells using leukaemia inhibitor factor (LIF)-free medium. Quantitative PCR revealed a decrease in Oct4 expression and an increase in Stra8 and Vasa mRNA in the EBs derived from iPS cells. iPS cell-derived EBs were induced by retinoic acid to differentiate into spermatogonial stem cells (SSCs), as evidenced by their expression of VASA, as well as CDH1 and GFR $\alpha 1$, which are markers of SSCs. Furthermore, these germ cells derived from iPS cells were transplanted into recipient testes of mice that had been pre-treated with busulfan. Notably, iPS cell-derived SSCs were able to differentiate into male germ cells ranging from spermatogonia to round spermatids, as shown by VASA and SCP3 expression. This study demonstrates that iPS cells have the potential to differentiate into late-stage male germ cells. The derivation of male germ cells from iPS cells has potential applications in the treatment of male infertility and provides a model for uncovering the molecular mechanisms underlying male germ cell development.

Asian Journal of Andrology (2012) 14, 574-579; doi:10.1038/aja.2012.3; published online 16 April 2012

Keywords: differentiation; induced pluripotent stem cells; male germ cells; retinoic acid; transplantation

\section{INTRODUCTION}

Primordial germ cells (PGCs) and spermatogonial stem cells (SSCs) form the founder population of male germ cells. Male gametes, specifically sperm, are directly derived from SSCs via spermatogenesis. Errors at any stage of spermatogenesis can result in subfertility or infertility, which are major public health issues affecting 10\%-15\% of couples. ${ }^{1}$ As an example, azoospermia is observed in $1 \%$ of the male population and in $10 \%-15 \%$ of infertile men. ${ }^{2}$ Furthermore, nonobstructive azoospermia, resulting from testicular failure, affects about $10 \%$ of infertile men and is diagnosed in $60 \%$ of azoospermic men.

Much progress has been made in the derivation of male germ cells from embryonic stem cells (ESCs). In mice, Hubner et al. ${ }^{3}$ first reported the successful derivation of male gametes from ESCs in vitro. Geijsen et al. ${ }^{4}$ isolated PGCs from mouse ESCs in vitro. In humans, the differentiations of germ cells from ESCs have also been demonstrated. ${ }^{3-7}$ However, there are some ethical problems surrounding the use of human ESCs. Furthermore, the sources of human ESCs are limited.

One of the major breakthroughs in stem cell biology was the establishment of induced pluripotent stem (iPS) cells from somatic cells by the retroviral transduction of one or several pluripotent genes, including Oct4, Sox2, c-Myc and Klf4. ${ }^{8,9}$ Notably, iPS cells have some advantages over human ESCs: (i) there are no ethical issues surrounding the use of human iPS cells; (ii) sources of human iPS cells are abundant; (iii) mature cells derived from patient iPS cells can be used for patientspecific cell therapy without immune rejection; and (iv) it may be feasible to obtain male germ cells from iPS cells derived from azoospermia patients to treat male infertility. Recent studies have demonstrated the feasibility of obtaining PGCs from iPS cells. iPS cells derived from mouse adult hepatocytes were able to be induced into PGCs. ${ }^{10}$ Vernet et al. ${ }^{13}$ also reported that human iPS cells could differentiate into PGCs when cocultured with human foetal gonadal cells. However, whether iPS cells can produce late-stage male germ cells has not been known yet.

The correct timing of sexual development has been demonstrated to require the early embryoid body (EB) expression of Stra-8, stimulated by retinoic acid (RA), and male-specific development proceeds this event. ${ }^{11}$ Furthermore, RA, an active derivative of vitamin A, regulates the timing of meiotic initiation in mice. ${ }^{11} \mathrm{RA}$ acts by binding to three nuclear retinoic acid receptors (RARs), including the RAR $\alpha, \beta$ and $\gamma$ isotypes. These receptors are ligand-dependent transcriptional regulators that transduce the RA signal. ${ }^{11,12}$ From neonatal development

${ }^{1}$ Renji Hospital, Sperm Development and Genetics Laboratory, Shanghai Human Sperm Bank, Shanghai Institute of Andrology, Department of Urology, Shanghai Jiao Tong University School of Medicine, Shanghai 200001, China; ${ }^{2}$ Bio-X Institutes, Key Laboratory for the Genetics of Developmental and Neuropsychiatric Disorders, Shanghai Jiao Tong University, Shanghai 200240, China; ${ }^{3}$ Clinic Stem Cell Research Center, Renji Hospital, Shanghai Jiao Tong University School of Medicine, Shanghai 200127, China and ${ }^{4}$ Department of Biochemistry and Molecular and Cellular Biology, Georgetown University Medical Center, Washington, DC 20057, USA

* These authors contributed equally to this work.

Correspondence: Professor Z Li (doc.zheng.li@gmail.com) and Professor Z He (zupinghe@gmail.com)

Received: 16 September 2011; Revised: 22 December 2011; Accepted: 15 January 2012; Published online: 16 April 2012 
to adulthood, each RAR is expressed in a specific cell type of the seminiferous epithelium: RAR $\alpha$ is expressed in Sertoli cells, RAR $\beta$ is expressed in round spermatids, and RAR $\gamma$ is expressed in type A spermatogonia. ${ }^{13}$ A recent study indicates that RA stimulates downstream gene expression in male testes after birth ${ }^{14}$ and that RA could be used to induce ESCs or iPS cells to differentiate into PGCs. Spermatogenesis is a very complex process that includes three key stages: mitosis, meiosis and spermiogenesis. In in vitro studies, inducing spermatogonial stem cells to undergo meiosis or obtaining spermatozoa has been proven difficult. In the current study, we combined in vitro differentiation and in vivo transplantation to obtain late-stage male germ cells. We used RA to promote the differentiation of iPS cells into PGCs and SSCs in vitro, as evidenced by the finding that iPS cells were positive for VASA and CDH1, respectively. After induction, iPS cell-derived germ cells were transplanted into the seminiferous tubules of busulfan-treated mice, and these cells further differentiated into late-stage male germ cells. This study thus provides a platform for exploring the molecular mechanisms underlying spermatogenesis and raises the possibility of using male germ cells derived from patientderived iPS cells in the treatment of male infertility.

\section{MATERIALS AND METHODS}

\section{Mouse iPS cells and culture}

A mouse iPS cell line (Tg-EGFP-miPS11.1; 40, XY) was donated by Professor Ying Jin (Shanghai Jiao Tong University School of Medicine, Shanghai, China). As previously described, iPS cells were generated from neural progenitor cells from enhanced green fluorescent proteins-transgenic C57BL/6J mice via retroviral transfer of human transcription factors Oct4/Sox $2 / \mathrm{Klf} 4 / \mathrm{C}-\mathrm{Myc}$, and this cell line has been demonstrated to be germline-competent. ${ }^{15}$ Mouse iPS cells were cultured in high glucose Dulbecco's modified Eagle's medium supplemented with $15 \%$ Fetal bovine serum, $0.1 \mathrm{mmol}^{-1}$ non-essential amino acids, $2 \mathrm{mmol} \mathrm{l}^{-1}$ L-glutamine, $0.1 \mathrm{mmol} \mathrm{l}^{-1}$ 2-mercaptoethanol, $100 \mathrm{U} \mathrm{ml}^{-1}$ penicillin, $100 \mathrm{mg} \mathrm{ml}^{-1}$ streptomycin and leukaemia inhibitor factor (LIF), on a feeder layer of mouse embryonic fibroblast in gelatinized dishes. The cells were passaged every $2-3$ days.

\section{Differentiation of iPS cells in vitro by RA induction}

After 2-3 days in culture, iPS cells were suspended in LIF-free medium to form EBs as described previously. ${ }^{16}$ iPS cell-derived EBs were induced to differentiate into male germ cells in vitro by treating cells with $10^{-6} \mathrm{~mol} \mathrm{l}^{-1} \mathrm{RA}$, and iPS-derived EBs were collected for subsequent quantitative PCR ( $\mathrm{qPCR}$ ) and immunocytochemical analysis. iPS-derived EBs that had not received RA treatment served as a negative control.

qPCR analysis of iPS cell-derived cells after RA treatment

Total RNA was extracted from iPS cell-derived cells using Trizol, and reverse transcription of purified RNA was performed using oligo(dT) priming and superscript II reverse transcription, according to the manufacturer's instructions (Invitrogen, Carlsbad, CA, USA). PCR reactions were performed as described previously. ${ }^{17}$ Primer pairs for selected genes, which are listed in Table 1, including Oct4, Vasa, Dazl and Stra8, were designed, and PCR products were separated by electrophoresis on $1.2 \%$ agarose gels. The PCR reaction was as follows: $94{ }^{\circ} \mathrm{C}$ for $5 \mathrm{~min}$; 30 cycles of PCR at $94{ }^{\circ} \mathrm{C}$ for $30 \mathrm{~s}, 54{ }^{\circ} \mathrm{C}$ for $30 \mathrm{~s}$ and $72{ }^{\circ} \mathrm{C}$ for $1 \mathrm{~min}$; and $72{ }^{\circ} \mathrm{C}$ for $15 \mathrm{~min}$. For qPCR, SYBR Green master mix was added to each well of the PCR reactions $(10 \mu \mathrm{l}$ of SYBR Green, $6 \mu \mathrm{l}$ of water, $1 \mu \mathrm{l}$ of primers and $2 \mu \mathrm{l}$ of cDNA). qPCR reactions were as follows: 40 cycles at $95{ }^{\circ} \mathrm{C}$ for $10 \mathrm{~s}$ and $60{ }^{\circ} \mathrm{C}$ for $60 \mathrm{~s}$. qPCR data and relative quantification were analysed using the Bio-Rad CFX Manager. Following the $\Delta C_{\mathrm{t}}$ method, the threshold of cycle values was normalized against the threshold value of mouse Gapdh. Statistically significant differences $(P<0.05)$ were determined by a one-way ANOVA and Tukey post-test using SPSS statistical software (SPSS Inc., Chicago, IL, USA).

\section{Flow cytometry analysis}

Single-cell suspensions of iPS cell-derived EBs were collected by centrifugation at $300 \mathrm{~g}$ for $5 \mathrm{~min}$. Cells were stained with Stemgent phycoerythrin anti-mouse SSEA1 (Biolegend, San Diego, CA, USA) for $30 \mathrm{~min}$ at $4{ }^{\circ} \mathrm{C}$. Then, the cells were washed twice with phosphatebuffered saline and analysed with an FACSCalibur system (Becton, Dickinson \& Company, San Jose, CA, USA). For each sample analysed, an aliquot of cells was labelled with mouse IgG conjugated to phycoerythrin as an isotype control.

\section{Immunofluorescence analysis of colocalisation of GFP and VASA or CDH1 in iPS cell-derived cells}

Immunofluorescence analysis of colocalisation of GFP and VASA or CDH1 in iPS cell-derived cells was performed according to a previously described method. ${ }^{18}$ After RA induction, iPS cell-derived cells were fixed by $4 \%$ paraformaldehyde and permeabilized with $0.1 \%$ Triton X-100. The cells were blocked using 5\% bovine serum albumin and were incubated with rabbit polyclonal primary antibody against VASA or CDH1 (1:100; Ab-Cam Inc., Cambridge, MA, USA). iPS cell-derived cells were then incubated with goat anti-rabbit secondary antibody and finally mounted in Vectashield mounting medium (Vector Laboratories, Inc., Burlingame, CA, USA). Immunofluorescence imaging was photographed using a TCS-SP-MP confocal microscope (Leica Corp., Deerfield, IL, USA).

\section{Busulfan-treated male ICR mice and transplantation of iPS cell-derived germ cells}

Male ICR mice of 6-8 weeks old (Shanghai Laboratory Animal Center, Chinese Academy of Sciences, Shanghai, China) were treated with busulfan (Sigma, St Louis, MO, USA) at $40 \mathrm{mg} \mathrm{kg}^{-1}$ body weight to deplete male germ cells in the testes. Mice were maintained in a specific pathogen-free environment. Animal welfare and experimental procedures were carried out strictly in accordance with the care and use of laboratory animals and the related ethical regulations of Shanghai Jiao Tong University (CULARER-SJTU). The recipient mice were transplanted with $20 \times 10^{6}$ cells ml $^{-1}$ iPS cell-derived cells in Dulbecco's modified Eagle's medium. Approximately $15 \mu \mathrm{l}$ of iPS cell-derived germ cells were transplanted into the seminiferous tubules of one testis via the efferent duct, and the testis without cell transplantation served

Table 1 Quantitative real-time PCR (qPCR) gene primers

\begin{tabular}{lll}
\hline Gene & \multicolumn{1}{c}{ Forward primer } & \multicolumn{2}{c}{ Reverse primer } \\
\hline Oct4 & 5'-ATCTGCTGAAGCAGAAGAGG-3' & 5'-GGTTCTCATTGTTGTCGGCT-3' \\
Vasa & 5'-GGATCCGAACCTGAAGCTATCATGGGA-3' & 5'-GTCGACCCATGATTCGTCATCAACTGG-3' \\
Dazl & 5'-TTCAGGCATATCCTCCTTATC -3' & 5'-ATGCTTCGGTCCACAGACTTC-3' \\
Stra8 & 5'-CAACCTGCAAGATGGGAATC-3' & 5'-CTTCAGCATCTGGTCCAACA-3' \\
\hline
\end{tabular}


as a control. Five weeks after transplantation, the testes of the recipient mice were collected for preparing frozen sections.

Immunofluorescence for colocalisation of GFP and VASA or SCP3 in testis with iPS cell-derived cell transplantation

Frozen sections from recipient mouse testes were incubated with an antibody to VASA or SCP 3 at $4{ }^{\circ} \mathrm{C}$ overnight. After extensive washes in phosphate-buffered saline, the sections were incubated with fluorescein (FITC)-conjugated IgG. DAPI (4', 6' -diamidino-2-phenylindole) was used to stain the nuclei of the cells in the testes, and the sections were observed for epifluorescence using a TCS-SP-MP confocal microscope (Leica Corp.). VASA expression in normal adult mouse testes served as a positive control.

\section{RESULTS}

Generation of EBs from iPS cells in vitro

Mouse iPS cells were cultured on an mouse embryonic fibroblast feeder layer with conditioned medium containing LIF (Figure 1a and b). After 2 or 3 days in culture, iPS cells were suspended in LIFfree medium to generate the EBs (Figure 1c and d). Under immunofluorescence microscopy, GFP expression was observed in iPS cells (Figure 1e) and in iPS cell-derived EBs (Figure 1f).

\section{Differentiation of iPS cell-derived EBs into male germ cells in vitro by RA induction}

iPS cell-derived EBs were induced by RA to differentiate into male germ cells in vitro. qPCR revealed that the expression of Oct4, which is a marker for ESCs and iPS cells, was decreased in iPS cell-derived EBs

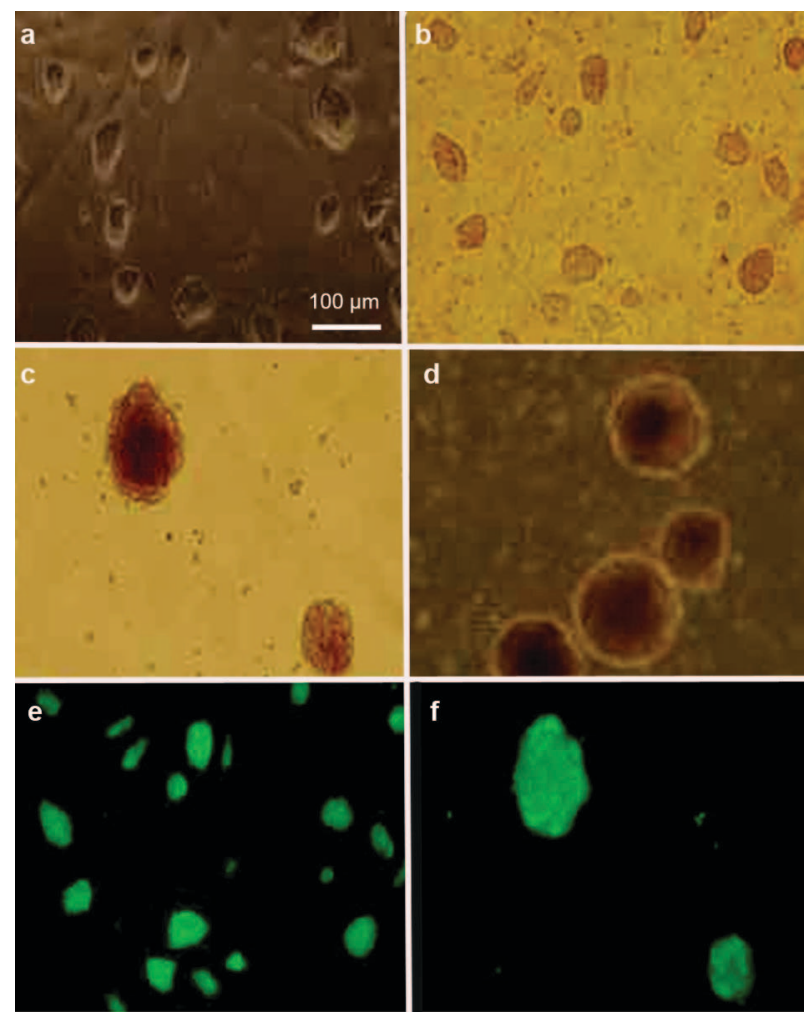

Figure 1 Morphological characterisation and GFP expression of iPS cells and iPS cell-derived EBs. Phase-contrast microscopy showed the morphological characteristics of iPS cells (a, b) and iPS cell-derived EBs (c, d). Immunofluorescent microscopy revealed GFP expression in iPS cells (e) and iPS cell-derived EBs (f). EB, embryoid body; GFP, green fluorescent protein; iPS, induced pluripotent stem. following RA induction compared with the cells without RA treatment (Figure 2 and Supplementary Figure 1). In contrast, the expression of Stra 8 was enhanced in iPS cell-derived EBs with RA induction compared with the cells without RA treatment (Figure 2 and Supplementary Figure 1). Although Dazl expression was not induced by RA in iPS cell-derived EBs, the expression of Vasa, a hallmark for male germ cells, was enhanced in iPS cell-derived EBs with RA induction compared with the cells without RA treatment (Figure 2 and Supplementary Figure 1), suggesting that RA induced iPS cellderived EBs to differentiate into germ cells in vitro.

Differentiation of iPS cell-derived EBs into spermatogonial stem cells in vitro by RA induction

After RA induction for 3 days, iPS cell-derived EBs were further analysed by immunofluorescence. Flow cytometry analysis of GFP and SSEA1 showed that the peak ratio (about 40\%) appeared on the third day after RA induction (Supplementary Figure 2), indicating that iPS cells had differentiated into PGCs. Confocal microscopy showed the colocalisation of GFP and VASA, which are markers for germ cells, in iPS-derived cells with RA induction (Figure 3). We further revealed that CDH1 and GFR $\alpha 1$, which are markers for SSCs, ${ }^{19}$ were coexpressed with GFP in the RAinduced iPS-derived cells (Figure 4 and Supplementary Figure 3), suggesting that RA induces iPS cells into SSCs in vitro. However, we did not find an increase in the expression of SCP3, which is a hallmark for spermatocytes, in iPS-derived cells with RA treatment (data not shown), indicating that these iPS-derived cells did not differentiate into spermatocytes in vitro.

Transplantation of iPS-derived cells into the seminiferous tubules of mice following busulfan treatment

Busulfan-treated mice are excellent recipients for germ cell transplants. Male germ cells were found to be depleted in the seminiferous tubules of mice treated with busulfan (Figure 5a and b) compared with the wild-type mice (Figure 5c). The germ cells, including PGCs and SSCs, derived from iPS-derived cells, were transplanted into the seminiferous tubules of mice following busulfan treatment (Figure 5d). In our experiments, all iPS cells were firstly induced to differentiate into EBs and SSCs and lose their pluripotency; hence, teratoma formation was infrequently observed.

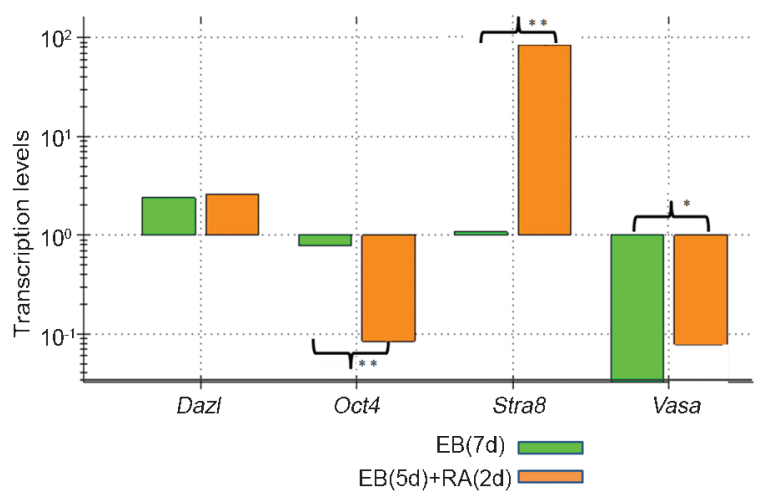

Figure 2 qPCR showing the transcription levels of the genes Dazl, Oct4, Stra8 and Vasa in iPS cell-derived EBs with or without RA treatment, compared with the control group $(\mathrm{EB}(7 \mathrm{~d}))(* P<0.05, * * P<0.01)$. EB, embryoid body; iPS, induced pluripotent stem; qPCR, quantitative PCR; RA, retinoic acid. 

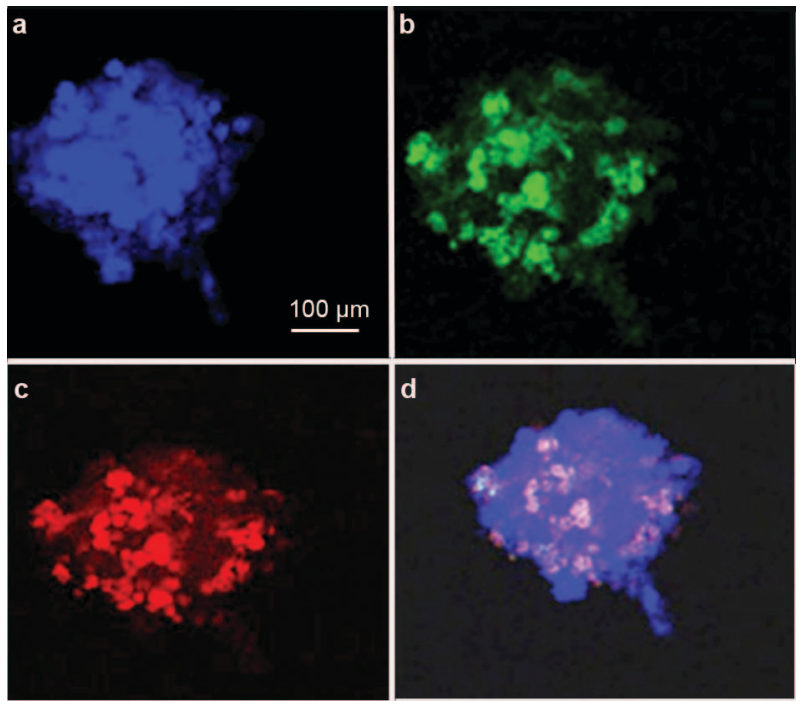

Figure 3 Confocal microscopy revealing the colocalisation of DAPI(a,d), VASA $(\mathbf{c}, \mathbf{d})$ and GFP (b,d) in iPS cell-derived cells exposed to RA in vitro. DAPI, 4', 6'diamidino-2-phenylindole; GFP, green fluorescent protein; iPS, induced pluripotent stem; RA, retinoic acid; VASA, DEAD box protein 4.

Colocalisation of VASA or SCP3 and GFP in the testes of mice with transplantation of iPS cell-derived germ cells

Five weeks after transplantation of iPS cell-derived SSCs, confocal microscopy showed the colocalisation of the GFP and VASA or SCP3, which are markers of male germ cells expressed from the late migration stage to the post-meiotic stage, ${ }^{10,20}$ in male germ cells from spermatagonia to round spermatids (Figure 6, top panel and Supplementary Figure 4), suggesting that the germ cells derived from iPS cells differentiated into late-stage male germ cells in vivo. We also observed the colocalisation of GFP and SCP3 in the recipient testis (Figure 7), further indicating that the germ cells derived from iPS cells differentiated into spermatocytes in vivo.
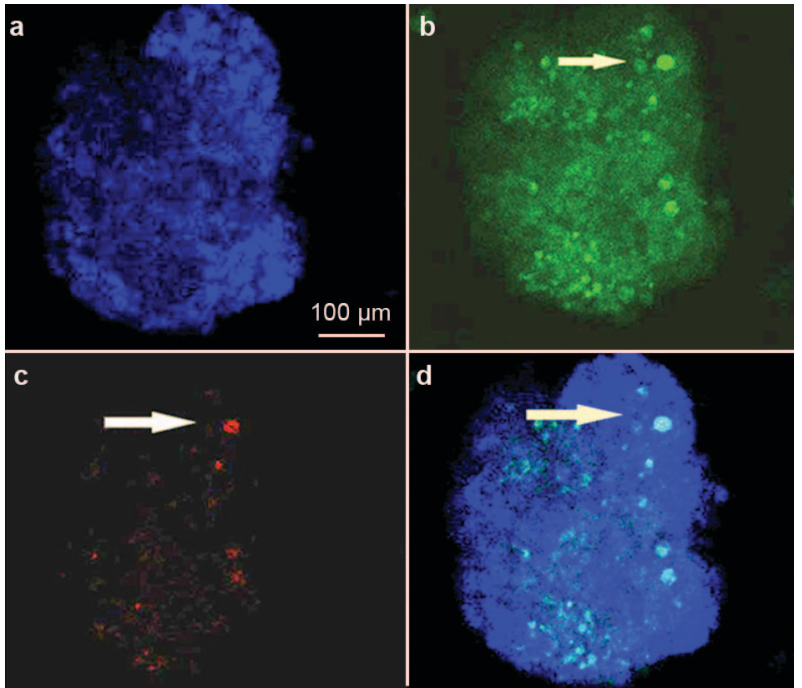

Figure 4 Confocal microscopy showing the colocalisation of DAPI(a,d), CDH1 $(\mathbf{c}, \mathbf{d})$ and GFP $(\mathbf{b}, \mathbf{d})$ in iPS cell-derived cells exposed to RA in vitro. $\mathrm{CDH} 1$, cadherin-1; DAPI, 4',6'-diamidino-2-phenylindole; GFP, green fluorescent protein; iPS, induced pluripotent stem; RA, retinoic acid.

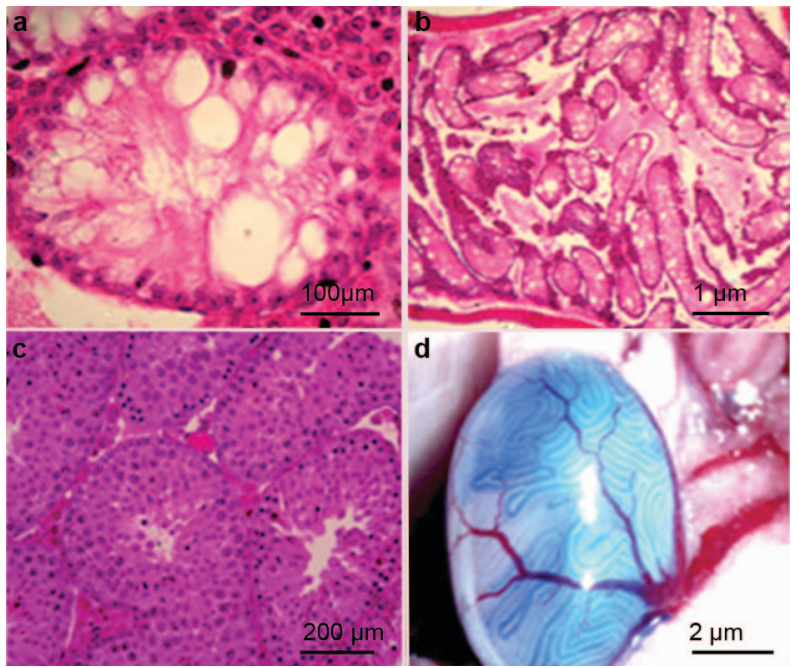

Figure 5 H\&E staining showing the morphology of mouse testes with busulfan treatment $(\mathbf{a}, \mathbf{b})$ or without busulfan treatment $(\mathbf{c}, \mathbf{d})$. Transplantation into mouse seminiferous tubules of SSCs derived from iPS cells through RA induction. iPS, induced pluripotent stem; RA, retinoic acid; SSC, spermatogonial stem cell.

\section{DISCUSSION}

iPS cells are pluripotent and therefore have the ability to differentiate into various types of cells that could be used for cell transplantation and tissue engineering in reproductive and regenerative medicine. However, few studies have examined the ability of iPS cells to differentiate into late-stage male germ cells. iPS cells from mice and humans have been shown to generate PGCs. ${ }^{10,21}$ Thus far, there is no perfect protocol for directing iPS cells towards the male gamete fate in vitro. Here, we demonstrated that iPS cells can produce latestage male germ cells.

Because inducing iPS cells to differentiate into the later stages of male germ cells in vitro is difficult, we employed an approach that combines in vitro differentiation and in vivo transplantation. First, we obtained EBs from iPS cells using LIF-free medium. The phenotypes of the EBs derived from iPS cells were verified by a decrease in Oct4 expression, which is a marker for pluripotent stem cells, and an increase in Stra8 and Vasa mRNA. Stra8 is a target gene of RA, and we found that the transcription of Stra 8 was significantly upregulated by RA in iPS cell-derived EBs compared with the cells that were not exposed to RA. Second, the EBs derived from iPS cells were induced by RA to differentiate into PGCs and SSCs, as evidenced by their expression of SSEA1, VASA, CDH1 and GFR $\alpha 1$. Since RA can promote the progression of iPS cells towards the primordial germ cell fate, ${ }^{8,21}$ it was thus chosen as the induction agent in this study. Finally, the germ cells derived from iPS cells were transplanted into recipient mouse testes lacking male germ cells. Notably, iPS cellderived SSCs can differentiate further into male germ cells from spermatagonia to round spermatids, as shown by VASA and SCP3 expression. Previous studies have shown that VASA (also known as Mvh) is a specific marker for differentiating germ cells from the late migration stage to the post-meiotic stage, and VASA is regarded as the most reliable marker for germ cell differentiation from stem cells. ${ }^{10,20}$ Additionally, SCP3 is one of the meiosis-specific components of the synaptonemal complex. SCP3 appears in leptotene spermatocytes but is downregulated in late meiotic cells. ${ }^{18}$ In this study, we demonstrated that iPS cells have the potential to differentiate into the later stages of male germ cells. The function of these late-stage male germ cells, and 


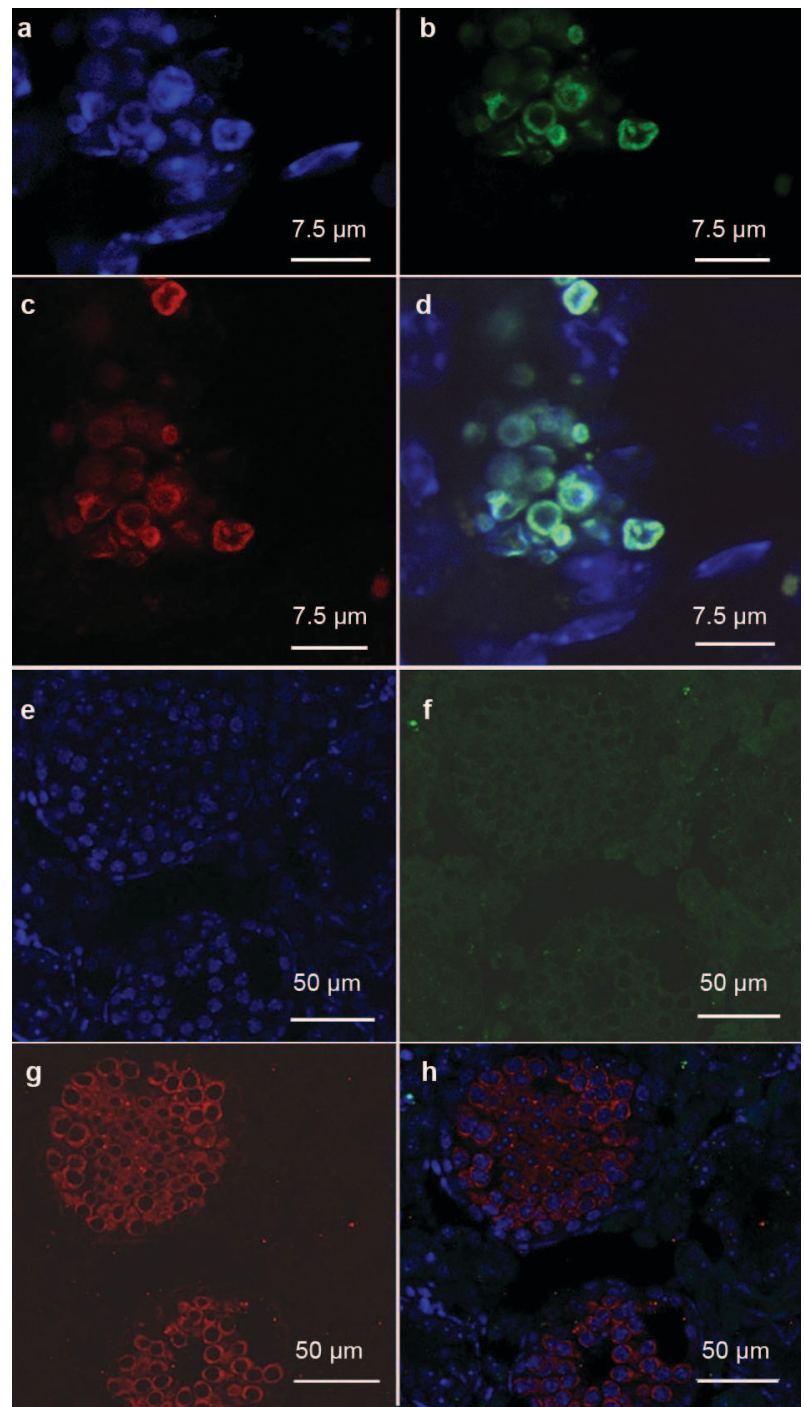

Figure 6 Confocal microscopy showing the colocalisation of VASA and GFP in recipient testes transplanted with germ cells RA-derived from iPS cells (a-d). VASA expression in normal mouse testes served as a positive control (e-h). DAPI, 4',6'-diamidino-2-phenylindole; GFP, green fluorescent protein; RA, retinoic acid; VASA, DEAD box protein 4

eventually the sperm derived from iPS cells, needs to be probed further by using intracytoplasmic sperm injection.

Azoospermia affects about $1 \%$ of the male population worldwide. Stem cell research is of great importance in treating male infertility. Nayernia et al. ${ }^{22}$ have reported the production of live mice from the sperm derived from ESCs in vitro; however, the mice died shortly after birth. The intrinsic testicular microenvironment, or the niche, including cell-cell conjunctions, specific hormones and growth factors, plays an essential role in the normal development of male germ cells. Thus, our approach of in vitro differentiation in combination with in vivo transplantation to generate late-stage male germ cells has great advantages over using in vitro differentiation only.

In summary, we demonstrated that iPS cells have the ability to differentiate into late-stage male germ cells. Our demonstration of the ability to generate male germ cells from iPS cells provides a paradigm for elucidating the mechanism of male germ cell development and has potential applications in the treatment of male infertility.

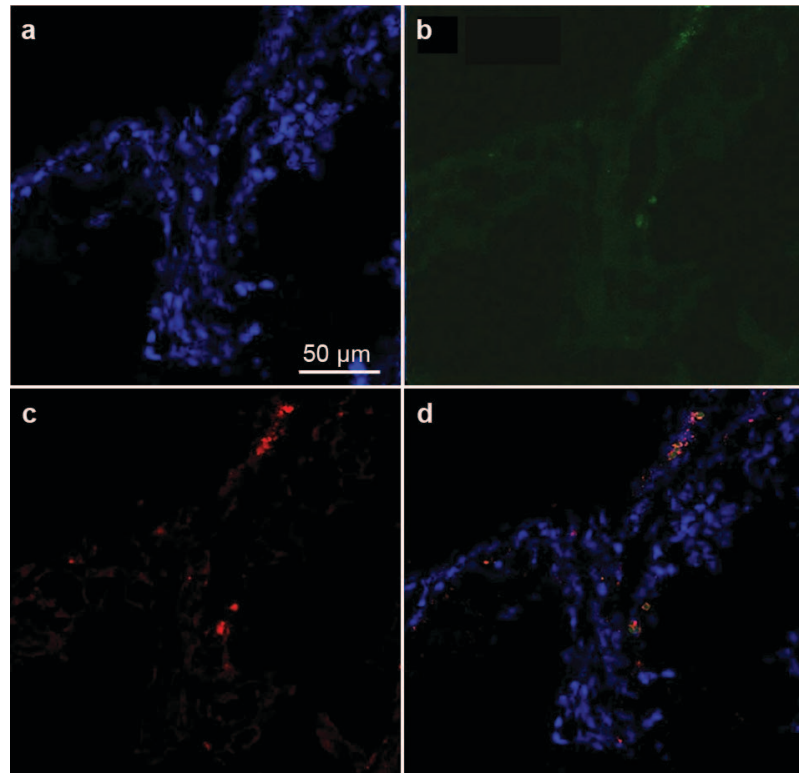

Figure 7 Confocal microscopy showing the colocalisation of DAPI(a,d), SCP3 $(\mathbf{c}, \mathbf{d})$ and GFP $(\mathbf{b}, \mathbf{d})$ in a recipient testis transplanted with germ cells RA-derived from iPS cells. GFP, green fluorescent protein; iPS, induced pluripotent stem; $\mathrm{RA}$, retinoic acid; SCP3, synaptonemal complex protein 3 .

\section{AUTHOR CONTRIBUTIONS}

ZL, ZH and XZG designed the experiments. YZ, PL, WZ, HD, RHT, SY, YN, LLZ and ZPS performed the research. YZ and HLH analysed the data and performed the statistical study. $\mathrm{YZ}, \mathrm{ZH}$ and $\mathrm{ZL}$ wrote the manuscript.

\section{COMPETING FINANCIAL INTERESTS}

The authors declare no competing financial interests.

\section{ACKNOWLEDGMENTS}

We greatly appreciate the kind donation of iPS cell line Tg-GFP-miPS11.1; 40,XY by Professor Ying Jin. This study was supported by the China National Key Project on Study of Disease Models and Mechanisms for Certain Major Diseases Based on iPS cell Technology (No. 2010CB945200), National Basic Research Program of China (No. 2011CB944504), Key Project of Shanghai Municipal Education Commission (No. 10ZZ70) and Nature Founding from Shanghai Jiao Tong University School of Medicine (No. 2008XJ017).

Supplementary Information accompanies the paper on Asian Journal of Andrology's website (http://www.nature.com/aja).

1 de Kretser DM, Baker HW. Infertility in men: recent advances and continuing controversies. J Clin Endocrinol Metab 1999; 84: 3443-50.

2 Willott GM. Frequency of azoospermia. Forensic Sci Int 1982; 20: 9-10.

3 Hubner K. et al. Derivation of oocytes from mouse embryonic stem cells. Science 2003; 300: 1251-6.

4 Geijsen N. et al. Derivation of embryonic germ cells and male gametes from embryonic stem cells. Nature 2004; 427: 148-54.

5 Aflatoonian B, Ruban L, Jones M, Aflatoonian R, Fazeli A et al. In vitro post-meiotic germ cell development from human embryonic stem cells. Hum Reprod 2009; 24: 3150-9.

6 West FD, Machacek DW, Boyd NL, Pandiyan K, Robbins KR et al. Enrichment and differentiation of human germ-like cells mediated by feeder cells and basic fibroblast growth factor signaling. Stem Cells 2008; 26: 2768-76.

7 Tilgner K, Atkinson SP, Golebiewska A, Stojković M, Lako M et al. Isolation of primordial germ cells from differentiating human embryonic stem cells. Stem Cells 2008; 26: 3075-85.

8 Okita K, Ichisaka T, Yamanaka S. Generation of germline-competent induced pluripotent stem cells. Nature 2007; 448: 313-7. 
9 Aoi T, Yae K, Nakagawa M, Ichisaka T, Okita K et al. Generation of pluripotent stem cells from adult mouse liver and stomach cells. Science 008321: 699-702.

10 Imamura M, Aoi T, Tokumasu A, Mise N, Abe K et al. Induction of primordial germ cells from mouse induced pluripotent stem cells derived from adult hepatocytes. Mol Reprod Dev 2010; 77: 802-11.

11 Koubova J, Menke DB, Zhou Q, Capel B, Griswold MD et al. Retinoic acid regulates sexspecific timing of meiotic initiation in mice. Proc Natl Acad Sci USA 2006; 103 2474-9.

12 Niederreither K, Dolle P. Retinoic acid in development: towards an integrated view. Nat Rev Genet 2008; 9: 541-53.

13 Vernet N, Dennefeld C, Guillou F, Chambon P, Ghyselinck NB et al. Prepubertal testis development relies on retinoic acid but not rexinoid receptors in Sertoli cells. EMBO J 2006; 25: 5816-25.

14 Bowles J, Koopman P. Retinoic acid, meiosis and germ cell fate in mammals Development 2007; 134: 3401-11.

15 Li C, Yu H, Ma Y, Shi G, Jiang J et al. Germline-competent mouse-induced pluripotent stem cell lines generated on human fibroblasts without exogenous leukemia inhibitory factor. PLOS ONE2009; 4: e6724.
16 Geijsen N, Horoschak M, Kim K, Gribnau J, Eggan K et al. Derivation of embryonic germ cells and male gametes from embryonic stem cells. Nature 2004; 427: 148-54.

$17 \mathrm{He}$ Z, Jiang J, Kokkinaki M, Dym M. Nodal signaling via an autocrine pathway promotes proliferation of mouse spermatogonial stem/progenitor cells through Smad2/3 and Oct-4 activation. Stem Cells 2009; 27: 2580-90.

18 Scherthan $\mathrm{H}$, Abend M, Müller K, Beinke C, Braselmann $\mathrm{H}$ et al. Centromere and telomere movements during early meiotic prophase of mouse and man are associated with the onset of chromosome pairing. J Cell Biol 1996; 134: 1109-25.

19 Tokuda M, Kadokawa Y, Kurahashi H, Marunouchi T. CDH1 is a specific marker for undifferentiated spermatogonia in mouse testes. Biol Reprod 2007; 76: 130-41.

20 Toyooka Y, Tsunekawa N, Akasu R, Noce T. Embryonic stem cells can form germ cells in vitro. Proc Natl Acad Sci USA 2003; 100: 11457-62.

21 Park TS, Galic Z, Conway AE, Lindgren A, van Handel BJ et al. Derivation of primordial germ cells from human embryonic and induced pluripotent stem cells is significantly improved by coculture with human fetal gonadal cells. Stem Cells2009; 27: 783-95.

22 Nayernia K, Nolte J, Michelmann HW, Lee JH, Rathsack K et al. In vitro-differentiated embryonic stem cells give rise to male gametes that can generate offspring mice. Dev Cell 2006; 11: 125-32. 